\title{
A model for ferrite-loaded transversely biased coaxial resonators
}

\author{
Acar, Öncel; Zhurbenko, Vitaliy; Johansen, Tom Keinicke
}

Published in:

2013 SBMO/IEEE MTT-S International Microwave and Optoelectronics Conference (IMOC)

Link to article, DOI:

10.1109/IMOC.2013.6646450

Publication date:

2013

Link back to DTU Orbit

Citation (APA):

Acar, Ö., Zhurbenko, V., \& Johansen, T. K. (2013). A model for ferrite-loaded transversely biased coaxial resonators. In 2013 SBMO/IEEE MTT-S International Microwave and Optoelectronics Conference (IMOC) IEEE. https://doi.org/10.1109/IMOC.2013.6646450

\section{General rights}

Copyright and moral rights for the publications made accessible in the public portal are retained by the authors and/or other copyright owners and it is a condition of accessing publications that users recognise and abide by the legal requirements associated with these rights.

- Users may download and print one copy of any publication from the public portal for the purpose of private study or research.

- You may not further distribute the material or use it for any profit-making activity or commercial gain

- You may freely distribute the URL identifying the publication in the public portal

If you believe that this document breaches copyright please contact us providing details, and we will remove access to the work immediately and investigate your claim 


\title{
A Model for Ferrite-Loaded Transversely Biased Coaxial Resonators
}

\author{
Öncel Acar ${ }^{1,2}$, Vitaliy Zhurbenko ${ }^{2}$, Tom K. Johansen ${ }^{2}$ \\ ${ }^{1}$ MTI Radiocomp \\ Krakasvej 17, 3400 Hillerød, Denmark \\ ${ }^{2}$ Technical University of Denmark \\ 2800 Kgs. Lyngby, Denmark \\ oncac/vz/tkj@elektro.dtu.dk
}

\begin{abstract}
This work describes a simple model for shortened coaxial cavity resonators with transversely biased ferrite elements. The ferrite allows the resonance frequency to be tuned, and the presented model provides a method of approximately calculating these frequencies to generate the tuning curve in short computation times. A test case resonator is specified, processed by the method, and constructed. Model predictions are compared to the measurement data as well as HFSS simulations. The model is also used for deducing maximum tuneability conditions on resonator parameters.
\end{abstract}

\section{Keywords—ferrite; tuneable; coaxial resonator; model.}

\section{INTRODUCTION}

Ferrite-loaded, magnetically tuneable coaxial cavity resonators operating in the radio frequency (RF) range are commonly used in particle accelerators [1-3]. The frequency of resonance in such cavities can be tuned by changing the DC magnetic field applied onto the ferrite elements. This is possible due to the bias-dependent magnetic behaviour of ferrite materials. With the introduction of the transverse bias configuration [2] where the bias field is parallel to the axis of the cavity, it was shown that the quality factors $(Q)$ of these resonators could approach those of unloaded cavities of the same geometry [4]. Despite their favourable features and utilization in practice, no simple way of calculating the resonance frequencies given the geometry and material properties seems to exist in former literature.

This work proposes a simple model that brings together the approximations for electromagnetic wave propagation in axially biased ferrite-loaded coaxial transmission lines, and the equivalent circuit representation of the shortened coaxial resonator type. The arising nonlinear equations are solved numerically in order to obtain the tuning characteristics of the cavity in short computation times. A sample cavity was constructed and measured for evaluation of the model's predictions. Furthermore, the same structure was simulated using Ansoft's HFSS [5] for comparison purposes. The advantages and disadvantages of the model are discussed.

\section{THE ANALYSIS}

The resonator, as mentioned earlier, is of the shortened coaxial type, meaning that it comprises a coaxial transmission line section that is short-circuited at one end, and terminated by a capacitor at the other. A possible way of realizing the capacitor in practice is to truncate the inner conductor of the coaxial line slightly before the end wall of the cavity, leaving an air gap. Inside the line, instead of a dielectric material, a ferrite sample is placed between the inner and the outer conductors. Figure 1 shows a drawing of such a structure together with its equivalent circuit model.

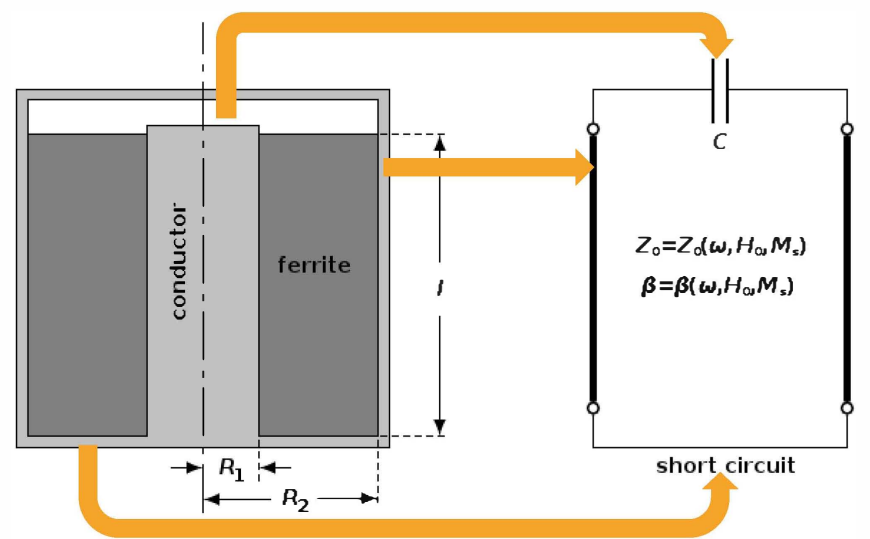

Fig. 1. Cross-sectional side view of the coaxial resonator and its equivalent circuit, with dimensions indicated, and corresponding parts matched.

In absence of the ferrite (i.e., for an air-filled coaxial line), such a structure would resonate at frequencies for which the susceptance of the capacitor and the port susceptance seen by the capacitor looking into the coaxial line add up to zero. This condition applies just the same way when the ferrite is present. However, the changes occurring in the wave propagation along the line need to be accounted for in the latter case. Assuming the line axis to be $z$-directed, when the ferrite is biased axially to saturation by a magnetic field $H_{0}$, it exhibits gyrotropic characteristics with a permeability tensor of the Cartesian form [6, p. 457]

$$
\bar{\mu}=\mu_{0}\left[\begin{array}{ccc}
\mu & j \kappa & 0 \\
-j \kappa & \mu & 0 \\
0 & 0 & 1
\end{array}\right],
$$

where $\mu_{0}$ is the magnetic permeability of the free space, and the tensor elements $\mu$ and $\kappa$ are functions of the DC bias field $H_{0}$

This work was conducted under the sponsorship of MTI Radiocomp. 
and the saturation magnetisation of the ferrite $4 \pi M_{\mathrm{s}}$ as given in the reference.

It has previously been shown that under this bias condition, quasi-TEM mode electromagnetic waves can propagate along the line [7] by analogy to the case of parallel plane waveguides which was presented by Suhl and Walker [8]. The propagation constant for the mentioned mode is approximately given by

$$
\beta\left(M_{\mathrm{s}}, H_{0}, \omega\right)=\omega \sqrt{\frac{\mu^{2}-\kappa^{2}}{\mu}} \epsilon,
$$

which essentially means that the propagating mode perceives an equivalent permeability of

$$
\mu_{\mathrm{e}}=\frac{\mu^{2}-\kappa^{2}}{\mu} \text {. }
$$

Here $\epsilon$ represents the dielectric permittivity of the ferrite, and $\omega$, the angular frequency of the electromagnetic wave. Henceforth, the characteristic impedance of the coaxial line can be written as

$$
Z_{0}\left(R_{1}, R_{2}, M_{\mathrm{s}}, H_{0}, \epsilon, \omega\right)=\frac{1}{2 \pi} \sqrt{\frac{\mu_{\mathrm{e}}}{\epsilon}} \ln \frac{R_{2}}{R_{1}},
$$

which can then be used for expressing the port susceptance seen by the capacitor looking into the line by involving the geometrical parameters of the coaxial line:

$$
B_{\mathrm{TL}}\left(R_{1}, R_{2}, l, M_{\mathrm{S}}, H_{0}, \epsilon, \omega\right)=-\frac{1}{Z_{0}} \cot (\beta l) .
$$

Stating the resonance condition now results in a closed form equation in $H_{0}, M_{\mathrm{s}}, R_{1}, R_{2}, l, C$ and $\omega$ :

$$
B_{\mathrm{TL}}\left(R_{1}, R_{2}, l, M_{\mathrm{s}}, H_{0}, \epsilon, \omega\right)+\omega C=0 .
$$

Equation 6 is central to the presented model for the resonator, which may be solved for one unknown parameter given the others. Below, two particular applications involving the solution of this equation are described further.

\section{A. A Test Case Solution and Evaluation}

For a certain ferrite material, cavity dimensions, and capacitive loading, Equation 6 is solved for $\omega$. Due to its nonlinearity and complexity as a function of $\omega$, the solution is sought numerically using the free software package GNU Octave [9]. As a test case, the set of cavity parameters seen in Table 1 is fed in to the equation.

TABLE I. TEST CASE RESONATOR PARAMETERS

\begin{tabular}{ccccccc}
\hline \multicolumn{3}{c}{ Ferrite properties } & \multicolumn{3}{c}{ Cavity measures } & Load \\
\hline $4 \pi M_{\mathrm{s}}(\mathrm{G})$ & $\Delta H(\mathrm{Oe})$ & $\epsilon_{0}$ & $R_{1}(\mathrm{~mm})$ & $R_{2}(\mathrm{~mm})$ & $l(\mathrm{~mm})$ & $C(\mathrm{pF})$ \\
\hline 1050 & 7 & 15 & 6 & 16 & 22 & 0,5 \\
\hline
\end{tabular}

As seen in Table 1, the ferrite material is intentionally selected to have a very small ferromagnetic resonance line width $(\Delta H)$ as that leads to lower losses, hence deviates less from the initial approximation of neglecting the losses.
The specified cavity was physically constructed and measured. In order to excite the cavity, an SMA connector was placed on its side wall, and the inner conductor of the connector was extended into the cavity space. The intrusion was kept at $1 \mathrm{~mm}$ to avoid a strong coupling that could perturb the quasi-TEM behaviour too much. The connector pin intrusion obviously required that the ferrite sample could not extend radially all the way to the outer conductor, so a $2 \mathrm{~mm}$ air gap had to be allowed. For this reason, to be able to provide a fair comparison, the calculated $\mu_{\mathrm{e}}$ values, as well as the known value of $\epsilon_{\mathrm{r}}$, were scaled by the ferrite fill factor to effective equivalent values. For the conductor losses to be as small as possible, the cavity surface was plated with a $10 \mu \mathrm{m}$ layer of silver. Figure 2 shows a photo of the constructed resonator and of the ferrite sample together with a view of the HFSS model of the cavity.

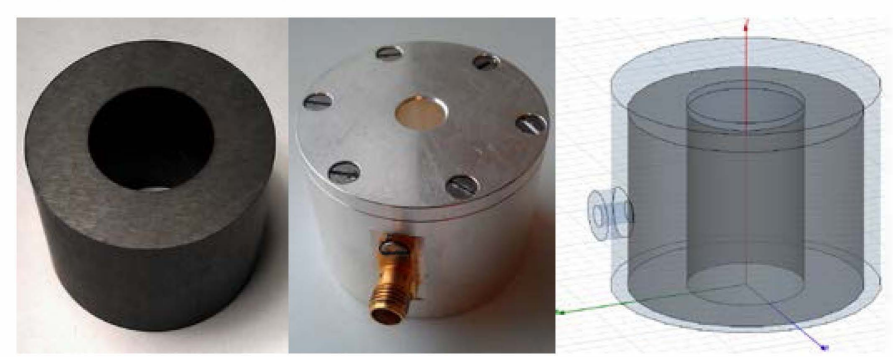

Fig. 2. Photographs of the ferrite sample and the constructed resonator, together with a view of the cavity's HFSS model.

The tuning curve of the resonator calculated numerically by the described method is plotted in Figure 3. The measurement results together with the data obtained from HFSS simulation of the same structure are also shown in the same figure for comparison.

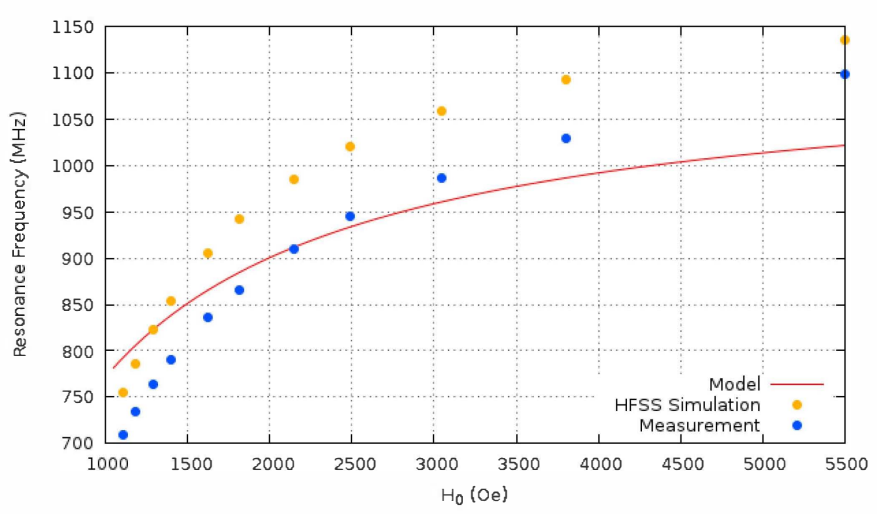

Fig. 3. Plot of the calculated tuning curve of the resonator together with the simulated and measured data for a selected set of bias levels.

The results are observed to lie within the $+11.6 \%$ to $-7.2 \%$ error range with reference to the measured data. The full wave simulator also appears to have some error, however it seems to follow the curvature of the measured data points' locus in a closer fashion. It can be argued that neglecting the inhomogeneity of the bias field is one of the reasons for the error.

Although the method is not particularly accurate, building up from the basics, it seems to capture the general tendency of 
tuning characteristics of the resonator, which is in agreement with previous findings [2]. The calculations can therefore be regarded as a valid approximation. The merit of the method is believed rather to be in its simplicity and the consequent calculation speed it delivers. In HFSS, it is not possible to use the eigenmode solver for problems involving ferrites [10], possibly due to the presented nonlinearity. This means that the driven mode solver needs to be employed together with a frequency sweep. To capture a resonance with as high $Q$ as the current problem, the sweep step size needs to be kept small, corresponding to hours of calculation time on a personal computer with a $3-\mathrm{GHz}$ quad-core processor and $12 \mathrm{~GB}$ of random access memory. In contrast, the presented method converges to a solution for a given $H_{0}$ in less than $10 \mathrm{~ms}$ on the same computer. It is thought that the method could be useful as a primer and help reduce the time used by $3 \mathrm{D}$ simulators.

\section{B. Implications on the Tuning Range of the Resonator}

Besides calculating the resonance frequencies for a given cavity, it could also be interesting to solve Equation 6 to investigate the relation between the tuning range and other parameters than $H_{0}$ (for a given span of $H_{0}$ ). Two particular questions, for instance, could be how to select the ferrite material for a given geometry, or how much capacitive loading to use, in case it is desired to maximise the tuneability. To address either of these questions, a tuneability parameter must be defined. Assuming that there may be practical difficulties in generating high levels of $H_{0}$, one way of defining this tuneability figure could be based on comparison of the two resonance frequencies: when the ferrite is just saturated, and when $H_{0}$ reaches the mentioned practical limit:

$$
\text { tuneability }=\frac{f_{\max }-f_{\text {sat }}}{f_{\text {sat }}} .
$$

Here, $f_{\text {sat }}$ and $f_{\max }$ represent the resonance frequencies at the onset of saturation and maximum bias states, respectively. Being a dimensionless ratio, tuneability can be expressed in percentages.

As an example, taking the mentioned upper limit for $H_{0}$ to be $5000 \mathrm{Oe}$, and considering the test case resonator used in the previous section, the tuneability is plotted against the saturation magnetisation $4 \pi M_{\mathrm{s}}$ in Figure 3 . It is hence shown that ferrite materials with lower saturation magnetisation should be preferred if wider tuning ranges are targeted.

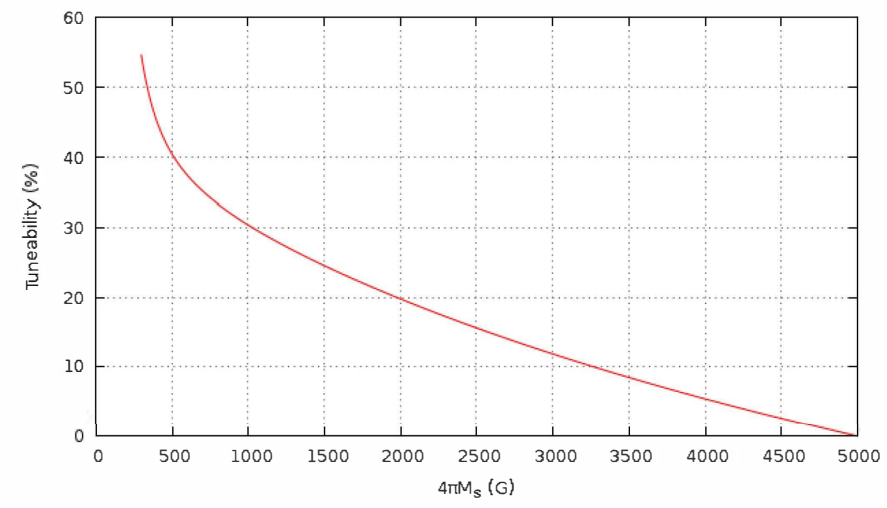

Fig. 4. Calculated tuneability versus saturation magnetisation for the test case resonator of the previous section.
The same approach could be followed to maximise the tuneability against any other parameter involved. This way, in design processes for instance, it becomes possible to very quickly obtain a good starting point before a big amount of time is committed to optimization through full wave 3D simulations.

\section{POSSIBLE IMPROVEMENTS TO THE MODEL}

The work presented here could certainly be extended to become more functional without compromising its simplicity and calculation speed. The first and the most important improvement to the presented model would be to take into account the magnetic losses in the ferrite. This could be possible by using the complex forms for $\mu$ and $\kappa$ in Equation 1 as stated in [6, p. 460], and could enable the model to predict the $Q$ values of the resonant modes in addition to the frequencies.

In some practical applications, the tuning range requirements are not as challenging and can be met by loading only a fraction of the cavity volume with a ferrite sample [2]. In such cases, for an expression that is equivalent to Equation 5 to be derived, the reflection at the interface of the ferrite-loaded line and the air-filled line needs to be taken into account. This requires a treatment of the boundary conditions imposed by the ferrite surface, and has previously been done by Brodwin and Miller [8]. In principle, the present work could be extended to cover cavities with arbitrary ferrite lengths by incorporating the cited results.

\section{CONCLUSION}

In this work, a simple model was presented for ferriteloaded, transversely biased coaxial resonators. The model was demonstrated to enable approximate solutions with very short calculation times (less than $10 \mathrm{~ms}$ on a personal computer) for the resonance frequencies such resonators, given the geometry measures of the cavity, the ferrite parameters and the bias field. The accuracy of the approximate solutions were evaluated and found to be within the $+11.6 \%$ to $-7.2 \%$ error margin. Although not as accurate as a full wave 3D solver, the method was seen to provide a good starting point that could reduce the time spent with more accurate tools.

It was also shown that the model could be employed in deducing requirements on resonator parameters in order to maximise the tuning range of the mentioned type of resonators. This was illustrated for the case of the ferrite's saturation magnetisation, and it was shown that in principle, lower saturation magnetisation levels will yield wider tuning ranges in transversely biased, ferrite-loaded coaxial cavity resonators.

\section{ACKNOWLEDGEMENT}

The authors would like to acknowledge MTI Radiocomp and the Technical University of Denmark for their support which enabled the present work. We would also like to express our thanks to Dr. Lara Scolari for her contributions.

\section{REFERENCES}

[1] G. Rakowsky, "RF accelerating cavities for AGS conversion," IEEE Transactions on Nuclear Science, vol. 14, issue 3, pp. 315-320, 1967. 
[2] L. M. Earley, H. A. Thiessen, R. Carlini, J. Potter, "A high-Q ferritetuned cavity," IEEE Transactions on Nuclear Science, vol. 30, issue 4 pp. 3460-3462, 1983.

[3] R. L. Poirier, T. Enegren, and C. Haddock. "Perpendicular biased ferrite tuned RF cavity for the TRIUMF KAON factory booster ring," in Proceedings of the 1989 Particle Accelerator Conference, pp. 171-173, 1989.

[4] W. R. Smythe et al., "RF cavities with transversely biased ferrite tuning," IEEE Transactions on Nuclear Science, vol. 32, issue 5, pp. 2951-2953, 1985.

[5] ANSYS® HFSS version 12.1, ANSYS Inc., available online: http://www.ansys.com/Products/Simulation+Technology/Electromagneti cs/High-Performance+Electronic+Design/ANSYS+HFSS
[6] D. M. Pozar, Microwave Engineering, $4^{\text {th }}$ ed., Wiley, 2011.

[7] M. E. Brodwin, D. A. Miller, "Propagation of the quasi-TEM mode in ferrite-filled coaxial line," IEEE Transactions on Microwave Theory and Techniques, vol. 12, issue 5, pp. 496-503, 1964.

[8] Suhl, Walker, "Topics in guided-wave propagation through gyromagnetic media," part 3, Bell System Technical Journal, vol. 33, issue 5, pp. 1133-1194, 1954.

[9] GNU Octave version 3.4.2, Octave community, available online: www.gnu.org/software/octave/

[10] ANSYS® HFSS version 12.1, help system, Eigenmode Solutions, ANSYS Inc. 